\title{
Phased array Doppler reflectometry at Wendelstein 7-X
}

Windisch, T.; Wolf, S.; Weir, G. M.; Bozhenkov, S. A.; Damm, H.; Fuchert, G.; Grulke, Olaf; Hirsch, M.; Kasparek, W.; Klinger, T.

Total number of authors:

15

Published in:

Review of Scientific Instruments

Link to article, DOI:

$10.1063 / 1.5039287$

Publication date:

2018

Document Version

Publisher's PDF, also known as Version of record

Link back to DTU Orbit

Citation (APA):

Windisch, T., Wolf, S., Weir, G. M., Bozhenkov, S. A., Damm, H., Fuchert, G., Grulke, O., Hirsch, M., Kasparek, W., Klinger, T., Lechte, C., Pasch, E., Plaum, B., Scott, E. A., \& Team, W-X. (2018). Phased array Doppler reflectometry at Wendelstein 7-X. Review of Scientific Instruments, 89(10), [10H115].

https://doi.org/10.1063/1.5039287

\section{General rights}

Copyright and moral rights for the publications made accessible in the public portal are retained by the authors and/or other copyright owners and it is a condition of accessing publications that users recognise and abide by the legal requirements associated with these rights.

- Users may download and print one copy of any publication from the public portal for the purpose of private study or research.

- You may not further distribute the material or use it for any profit-making activity or commercial gain

- You may freely distribute the URL identifying the publication in the public portal 


\section{Phased array Doppler reflectometry at Wendelstein 7-X}

T. Windisch, S. Wolf, G. M. Weir, S. A. Bozhenkov, H. Damm, G. Fuchert, O. Grulke, M. Hirsch, W. Kasparek,

T. Klinger, C. Lechte, E. Pasch, B. Plaum, E. A. Scott, and W7-X Team

Citation: Review of Scientific Instruments 89, $10 \mathrm{H} 115$ (2018); doi: 10.1063/1.5039287

View online: https://doi.org/10.1063/1.5039287

View Table of Contents: http://aip.scitation.org/toc/rsi/89/10

Published by the American Institute of Physics

\section{Articles you may be interested in}

Turbulence level effects on conventional reflectometry using 2D full-wave simulations

Review of Scientific Instruments 89, $10 \mathrm{H} 110$ (2018); 10.1063/1.5038756

First step toward a synthetic diagnostic for magnetic fluctuation measurements using cross-polarization scattering on DIII-D

Review of Scientific Instruments 89, $10 \mathrm{H} 113$ (2018); 10.1063/1.5036791

Using time-resolved penumbral imaging to measure low hot spot $\mathrm{x}$-ray emission signals from capsule implosions at the National Ignition Facility

Review of Scientific Instruments 89, 10G111 (2018); 10.1063/1.5037073

An eight-channel Doppler backscattering system in the experimental advanced superconducting tokamak Review of Scientific Instruments 88, 073504 (2017); 10.1063/1.4991855

Overview of the Wendelstein 7-X phase contrast imaging diagnostic

Review of Scientific Instruments 89, 10E105 (2018); 10.1063/1.5038804

Optimized quasi-optical cross-polarization scattering system for the measurement of magnetic turbulence on the DIII-D tokamak

Review of Scientific Instruments 89, 10H107 (2018); 10.1063/1.5035427

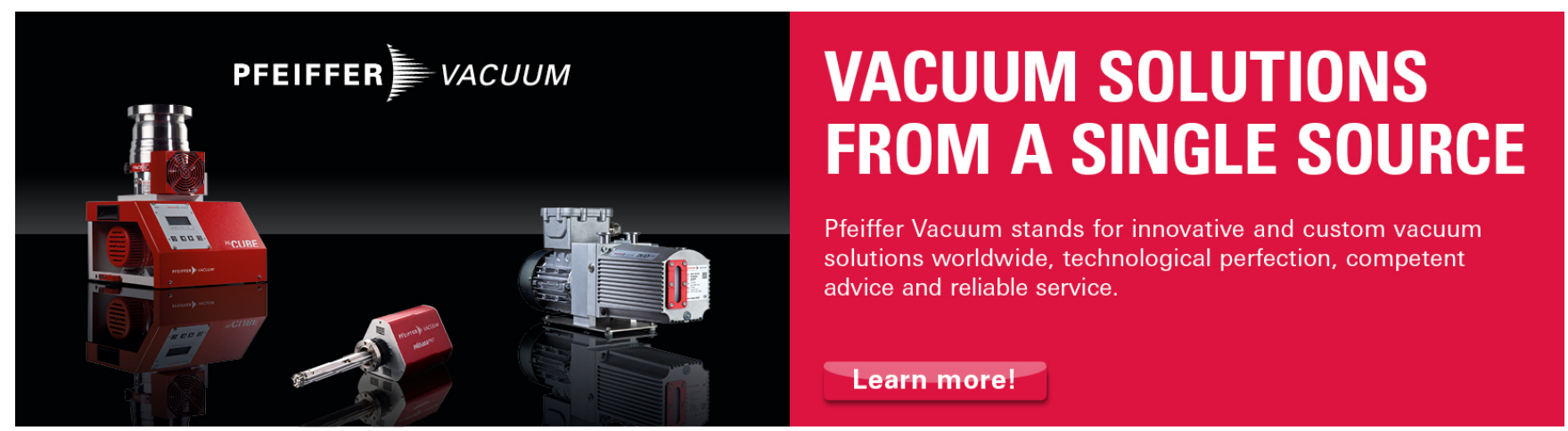




\title{
Phased array Doppler reflectometry at Wendelstein 7-X
}

\author{
T. Windisch, ${ }^{1, \text { a) }}$ S. Wolf, ${ }^{2}$ G. M. Weir,${ }^{1}$ S. A. Bozhenkov,${ }^{1}$ H. Damm, ${ }^{1}$ G. Fuchert, ${ }^{1}$ \\ O. Grulke,,$^{1,3}$ M. Hirsch, ${ }^{1}$ W. Kasparek, ${ }^{2}$ T. Klinger, ${ }^{1}$ C. Lechte, ${ }^{2}$ E. Pasch, ${ }^{1}$ B. Plaum, ${ }^{2}$ \\ E. A. Scott, ${ }^{1}$ and W7-X Team ${ }^{1, b)}$ \\ ${ }^{1}$ Max-Planck-Institute for Plasma Physics, Greifswald, Germany \\ ${ }^{2}$ Institut für Grenzflächenverfahrenstechnik und Plasmatechnologie, Universität Stuttgart, Stuttgart, Germany \\ ${ }^{3}$ Department of Physics, DTU, DK-2800 Lyngby, Denmark
}

(Presented 18 April 2018; received 7 May 2018; accepted 14 June 2018; published online 28 September 2018)

\begin{abstract}
A passive phased array Doppler reflectometry system has recently been installed in the Wendelstein$7 \mathrm{X}$ stellarator. In contrast to conventional Doppler reflectometry systems, the microwave beam can be steered on short time scales in the measurement plane perpendicular to the magnetic field in the range of $\pm 25^{\circ}$ without mechanical steering components. This paper characterizes the design and properties of the phased array antenna system and presents the first measurement results from the latest OP1.2a campaign. https://doi.org/10.1063/1.5039287
\end{abstract}

\section{INTRODUCTION}

In the last two decades, Doppler reflectometry became an established diagnostic technique to measure mean plasma flows and associated fluctuations in fusion devices with high spatial and temporal resolution. ${ }^{4,6}$ In contrast to normal incidence (conventional) reflectometry, the microwave beam is launched oblique to the flux surface with incident angle $\theta_{i}$. Density fluctuations aligned along the magnetic field $\left(k_{\perp} \gg k_{\|}\right)$corrugate the cut-off layer and thereby scatter the incident beam according to the Bragg condition $m k_{\perp}=k_{0}$ $\left(\sin \theta_{i}-\sin \theta_{m}\right.$ ) (slab geometry). For backscattering conditions $\left(\theta_{i}=-\theta_{m}=\theta\right)$, this reduces to $k_{\perp}=2 k_{0} \sin (\theta)$ such that a variation of the incident tilt angle $\theta_{i}$ allows us to measure complete fluctuation wavenumber spectra $S\left(k_{\perp}, r, t\right)$. The localization of the scattering origin near the cutoff and the scattering wavenumber are calculated using ray (beam) tracing techniques or full-wave simulations. ${ }^{9}$ The poloidal propagation of fluctuations introduces a Doppler shift of the scattered electric field, $\Delta \omega=k_{\perp} v_{\perp} \approx k_{\perp} E_{r} / B$, from which the radial electric field $E_{r}$ can be deduced if the phase velocity of the fluctuations is negligible, which is usually fulfilled. ${ }^{1}$ In recent devices, the incident angle $\theta$ is actively controlled by mechanical steering of plasma facing off-axis parabolic mirrors. ${ }^{3}$ The mechanical system introduces limits to the steering rate $(d \theta / d t)$ and might also be incompatible with the harsh environment of fusion reactors during long pulses operation and in-vessel space limitations. An alternative method to $\operatorname{control} \theta$ is a phased array antennae (PAA) system, which allows to steer the microwave beam without any movable parts. Such phased array systems are well known from airborne radar applications, e.g., for weather and space observation. ${ }^{2}$ A PAA system consists of multiple antenna elements which emit separate

\footnotetext{
Note: Paper published as part of the Proceedings of the 22nd Topical Conference on High-Temperature Plasma Diagnostics, San Diego, California, April 2018.

a)Electronic mail: thomas.windisch@ipp.mpg.de

b) See the author list of R. C. Wolf et al. Nucl. Fusion 57, 102020 (2017).
}

microwave signals. When the phase shift between neighboring elements vanishes or is a multiple of $2 \pi$, the far-field maximum is directed along the aperture (array) normal. By introducing a progressive phase shift in the individual signals with a delay on the order of fractions of a wavelength $\lambda_{0}$, the region of constructive interference can be steered such that a constructive signal appears at an angle to the array normal. The phase shift can either be produced by passive elements (e.g., different waveguide lengths of each element), or it can be actively controlled using phase shifters. For the application in fusion devices, a compact 32-element prototype operating in W-band $(75-110 \mathrm{GHz})$ has been designed, built, and successfully tested under lab conditions. ${ }^{14,18,19}$ A similar preceding system was built and installed on ASDEX Upgrade. ${ }^{19}$ This paper describes the implementation of the PAA system at the stellarator Wendelstein-7X $\mathrm{X}^{7}$ and presents first measurement results.

\section{DIAGNOSTIC SETUP}

The passive 32-element PAA has two components: a feed network and an H-plane sectoral horn array $(l=20 \mathrm{~cm})$ for beam shaping and side lobe suppression. ${ }^{14}$ The feed network [cf. Fig. 1(a)] consists of a helical WR10 waveguide delay line, produced by galvanically closing a waveguide channel milled into a CuCrZr core [cf. Fig. 1(b)]. It has one coupling element on each of its 32 turns. Only a tiny fraction of the power are coupled to the individual horns (typically $-30 \mathrm{~dB}$ ). Taking all 32 holes into account, $-15 \mathrm{~dB}$ of the input power is coupled to the antenna array such that the open ended helical waveguide channel needs a proper load to avoid reflections. Since the latter would strongly affect the internal phase relation, the system has been designed as a bistatic system, i.e., two separate PAA systems for launching (TX) and receiving (RX) have been manufactured. A glimpse of the bistatic invessel components are shown in Fig. 1(c), together with the antenna orientation and the measurement plane defined by the central ray indicated in blue. The in-vessel transmission line $(l \approx 1.5 \mathrm{~m})$ consists of fundamental WR10 waveguides 

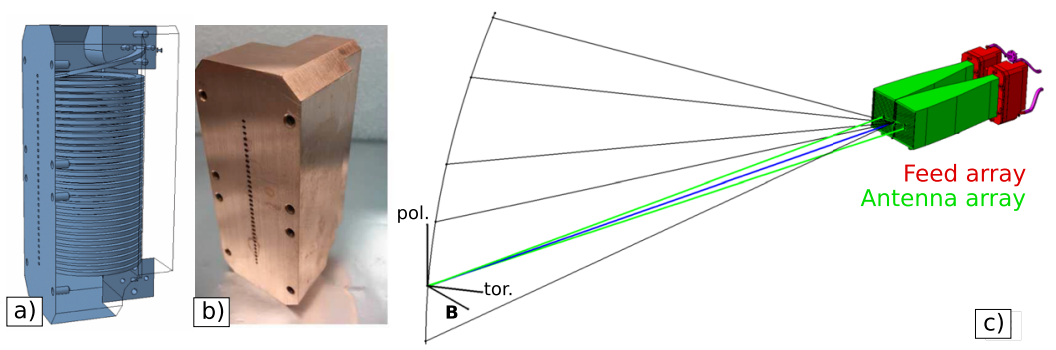

FIG. 1. (a) and (b) CAD and electroformed feed array with 32 elements (dimensions $110 \mathrm{~mm} \times 35 \mathrm{~mm}$ ) and (c) bistatic antennae setup (CAD).
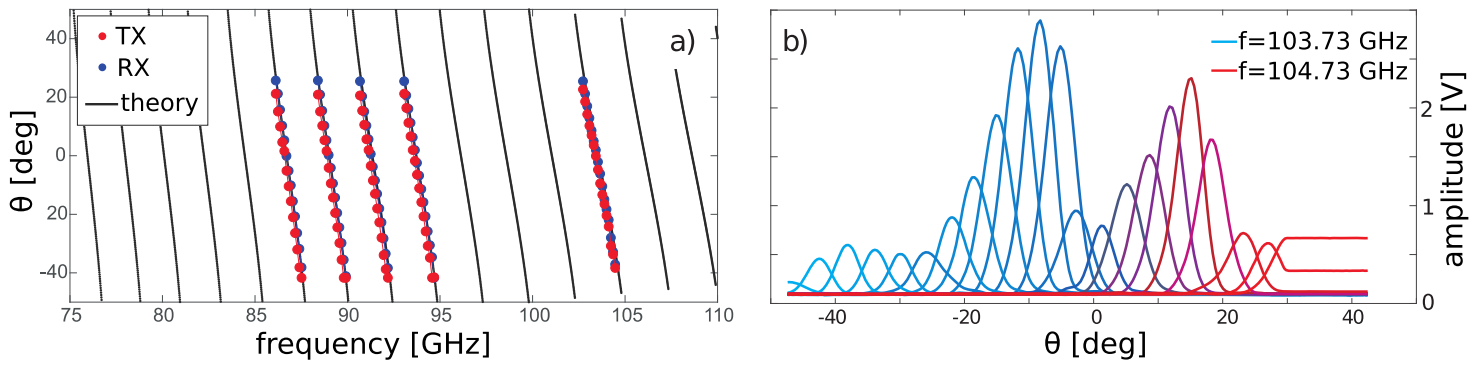

FIG. 2. (a) Measured (red and blue dots) and theoretical (solid) steering angle dependence $\theta(f)$. (b) Measured one-dimensional slices of the far-field antenna pattern ( $E$-plane, distance to antenna aperture $d r=45 \mathrm{~cm}$ ) for one steering sweep with central frequency $f_{c}=104.23 \mathrm{GHz}$.

connected to the input and output flanges of the feed array $(l \approx 30 \mathrm{~cm}$ ), oversized $d=4 \mathrm{~mm}$ circular waveguides, and tapers for the transition. The vacuum barrier is a $d=4 \mathrm{~mm}$ mica sheet window (thickness $100 \mu \mathrm{m}$ ). For reasons of $140 \mathrm{GHz}$ ECRH stray radiation compatibility, the feed array output is connected to an open ended WR10 stainless steel waveguide which provides sufficient attenuation of the residual signal $(20 \mathrm{~dB} / \mathrm{m})$ to avoid reflections. The ex-vessel transmission line $(l=22 \mathrm{~m})$ is made of oversized circular waveguides $(d=28 \mathrm{~mm})$ and optimized nonlinear tapers for the TE10 transition from $d=4 \mathrm{~mm}$ to $d=28 \mathrm{~mm}$. The overall attenuation of the ex-vessel transmission line is $7-8 \mathrm{~dB}$. This is mainly caused by nine mitre bends which are necessary to guide the transmission line from the torus into the measurement cabinet. The PAAs are driven by a frequency hopping reflectometer with $10 \mathrm{dBm}$ RF output and standard heterodyne $I Q$-detection.

The PAA is designed to cover at least a steering range of $-20^{\circ} \leq \theta \leq 20^{\circ}$. The slope $d \theta / d f$ is chosen to allow for steering sweeps around 15 central frequencies $f_{c}\left(\theta\left(f_{c}\right)=0\right)$ almost equally distributed across the entire W-band. The central frequencies are separated by $\approx 2 \mathrm{GHz}$. The theoretical steering angle dependence $\theta(f)$ is shown in Fig. 2(a) as black lines. Within a frequency variation of $f_{c} \pm 500 \mathrm{MHz}$, the design steering range is covered. The steering angle is given by $\theta=\arcsin \left(\Delta \phi \lambda_{0} /(2 \pi d)\right)$, where $\Delta \phi=-2 \pi L / \lambda_{g} \epsilon[-\pi, \pi]$ is the phase variation between neighboring coupling holes, $\lambda_{0}=c / f$ is the free space wavelength, and $\lambda_{g}=\lambda_{0} / \sqrt{1-\left(\lambda_{0} /(2 a n)\right)^{2}}$ is the waveguide wavelength with waveguide width $a$ and refractive index $n$. $L$ denotes the helix winding length $L=\sqrt{\left.(2 \pi(R-a / 2))^{2}+d^{2}\right)}$ for outer winding diameter $R$ and element spacing $d$. Machining imperfections lead to small deviations from the design parameters, which can alter the steering properties significantly. The deviations can only be characterized by measurements of the $\theta(f)$ dependence in the far-field. An example is shown in Fig. 2(a) for five central frequencies $f_{c}$. Both PAAs (TX and RX) have the same dependence which is essential for the bistatic operation. The black lines indicate the analytical expression with modified parameters. Across the frequency band, the slope $d \theta / d f$ changes due to the decreasing wavelength. The variation of the beam power within one steering sweep centered at $f_{c}=103.43$ is shown in Fig. 2(b). In the design steering range $-20^{\circ} \leq \theta$ $\leq 20^{\circ}$, the beam power exhibits $\sim 3-4 \mathrm{~dB}$ variations, which need to be taken into account when comparing the measured scattered power at different $\theta$. Especially at small steering

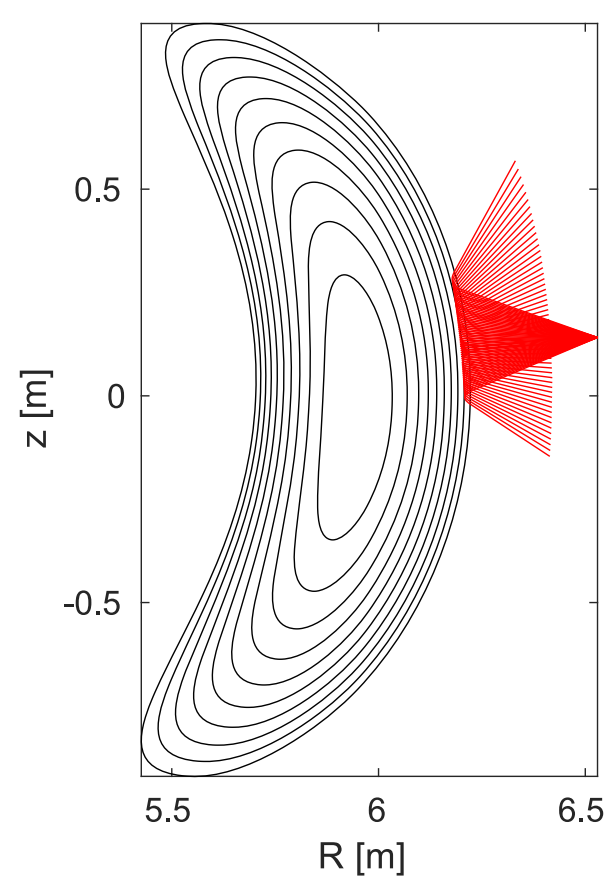

FIG. 3. Vacuum flux surfaces at $\phi=71^{\circ}$ (VMEC) with ray tracing trajectories. 
angles $|\theta|<3^{\circ}$, the power decreases significantly (open stopband ${ }^{15}$ ). A slight asymmetry between $\theta<0$ and $\theta>0$ is also observed.

At Wendelstein 7-X (av. major radius $R=5.5 \mathrm{~m}$ ) the bistatic PAA is located in the so-called bean shaped plane (cf. Fig. 3), in which the flux-surface elongation is maximum and the magnetic field scales approximately as $R^{-1}$. The large toroidal and poloidal curvatures, $\rho_{\text {tor }} \sim 6 \mathrm{~m}$ and $\rho_{\text {pol }} \sim 1 \mathrm{~m}$, respectively, are beneficial to achieve a good wavenumber resolution. ${ }^{10}$ The PAA is located $140 \mathrm{~mm}$ above the midplane and has a poloidal tilt angle offset of $-10^{\circ}$ to ensure good overlap with other reflectometer systems at the same port. ${ }^{16,17}$ Typical ray trajectories as calculated with the ray-tracing code TRAVIS ${ }^{11}$ are shown in Fig. 3 for the accessible steering range as red lines.

\section{MEASUREMENT RESULTS}

First, measurements with the PAA system at Wendelstein $7-\mathrm{X}$ have been performed in the latest campaign (operation phase 1.2a). The measurements presented here focus on one slow steering sweep with $f_{c}=86.85 \mathrm{GHz}$ (W7-X program id $171129016)$ and high resolution $(\Delta f=10 \mathrm{MHz}$ for consecutive frequency hopping plateaus). Each steering frequency plateau [cf. Fig. 4(b)] is sampled for $10 \mathrm{~ms}$ with $10 \mathrm{MHz}$ to enhance spectral resolution. During the steering sweep in the time interval $t=1500-3100 \mathrm{~ms}$, plasma density and ECRH

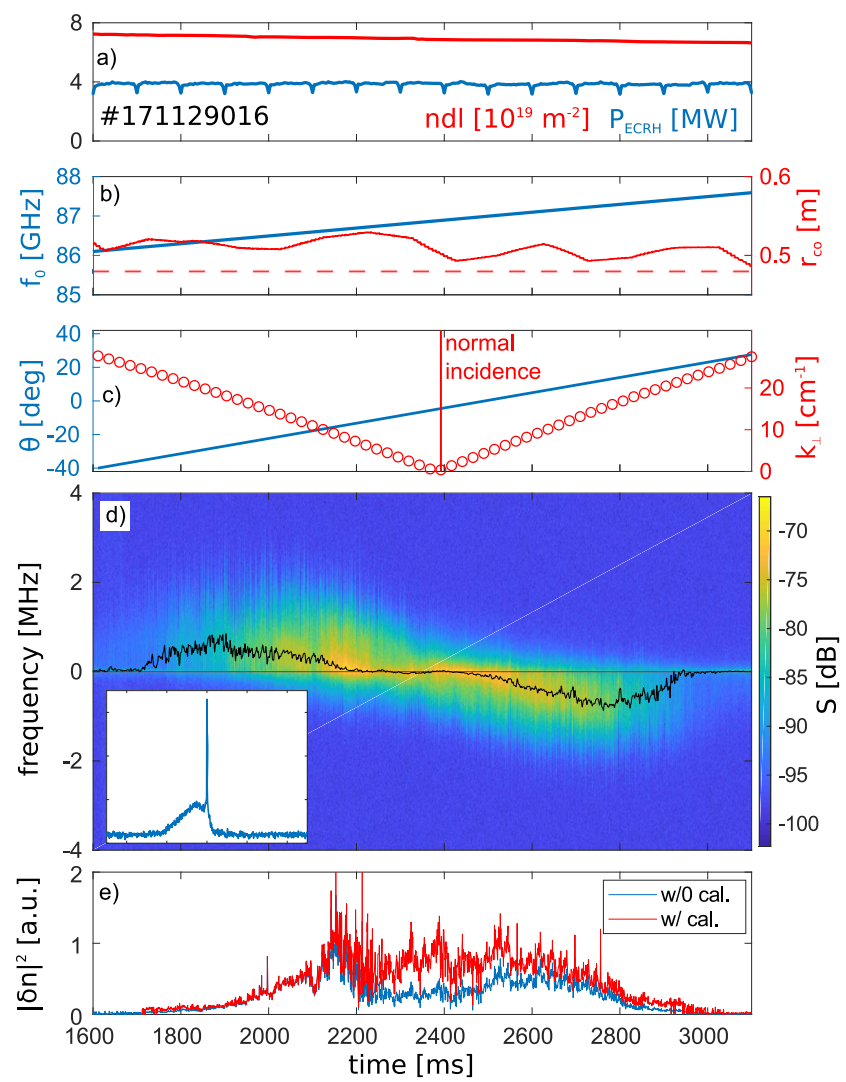

FIG. 4. Parameter overview for W7-X program id 171129016: (a) ECRH power (blue) and line integrated density (red), (b) PAA frequency (blue) and cut-off layer position $r_{c o}$ (red), (c) steering angle $\theta$ (blue) and $k_{\perp}$ (red), (d) Doppler spectrum with Doppler frequency obtained from Gaussian fit (solid black), and (e) measured scattered power (see text for details). heating power $P_{E C R H}$ are approximately constant [Fig. 4(a)]. $P_{E C R H} \approx=4 \mathrm{MW}$ and the line integrated density varies slightly between $7.2 \cdot 10^{19} \mathrm{~m}^{-2}$ and $6.6 \cdot 10^{19} \mathrm{~m}^{-2}$. Due to the high plasma density, the $X$-mode cutoff is outside the last closed flux surface (LCFS) in the scrape-off layer (SOL) region for all steering frequencies. A typical density profile obtained from the Thomson scattering system ${ }^{12}$ is shown in Fig. 5 for time instant $t=2600 \mathrm{~ms}$. The position of the LCFS, $r_{\text {eff }}^{L C F S}=0.48 \mathrm{~m}$ is indicated as a red line. The cutoff layer position $r_{c o}$ and the backscattering Bragg (fluctuation) wavenumber $k_{\perp}=-2 k_{0} N_{\perp}\left(N_{\perp}\right.$ denotes refractive index) are determined for the individual frequencies and steering angles from ray-tracing (TRAVIS) for the central ray [cf. Fig. 1(c)].

The incident beam power profile transverse to $k_{0}$ is shown in Fig. 6 at a distance of $d z=40 \mathrm{~cm}$ from the antenna aperture, which corresponds to the nominal distance to the cut-off layer. The beam profile is slightly elliptical with $1 / e^{2}$-widths $w_{H}=36.5 \mathrm{~mm}$ and $w_{E}=25.8 \mathrm{~mm}$ in the $\mathrm{H}$ - and E-plane, respectively. The tilt of the ellipse indicates the alignment of the measurement plane to the pitch angle $\tan (\alpha)=B_{z} / B_{\phi}$ $\approx 12.5^{\circ}$ of the ambient magnetic field in the cut-off layer region.

The temporal evolution of $r_{c o}$ is depicted in Fig. 4(b) as a red line (the dashed red line indicates the LCFS position). The distance of $r_{c o}$ to the LCFS is in the range of $2-3 \mathrm{~cm}$ and decreases linearly with time as expected from the lineaveraged density [cf. Fig. 4(a)]. The frequency of the $r_{c o}$ oscillations is set by the limited time resolution of the Thomson scattering system, which has a repetition rate of $100 \mathrm{~ms}$. For the time being, the further analysis is performed with linearly interpolated profiles in between the acquisition time of the Thomson scattering system. Time series of $\theta$ and $k_{\perp}$ are illustrated in Fig. 4(c). Due to viewing geometry of the PAA (cf. Sec. II), $\theta$ is not symmetric, i.e., $\theta \approx-5.5^{\circ}$ corresponds to normal incidence as indicated by the red solid line. The signal-to-noise ratio of the measured $I Q$-signal is poor, $P_{S / N} \approx 12 \mathrm{~dB}$. This is mainly caused by the small coupling efficiency between the feed array and the antenna array as already described in Sec. II. The spectrogram of the measured complex $I Q$-signal is illustrated in Fig. 4(d). For $\left|k_{\perp}\right| \lesssim 20 \mathrm{~cm}^{-1}$, a clear Doppler peak is observed. As $\theta$ crosses the flux-surface normal, the frequency of the Doppler peak changes the sign.

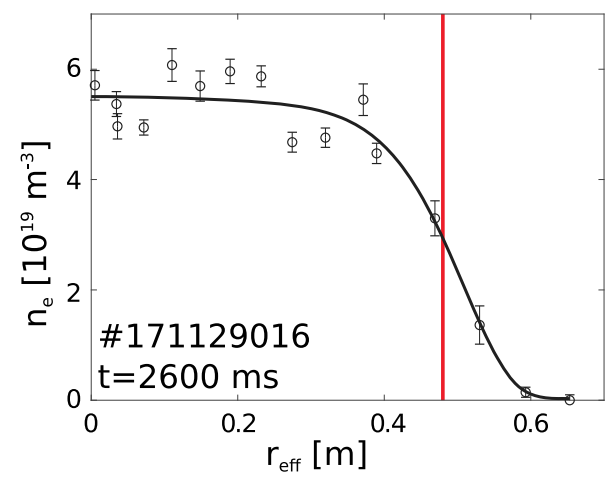

FIG. 5. Electron density profile measured with Thomson scattering at time instant $t=2600 \mathrm{~ms}$ (W7-X program id 171129016). 


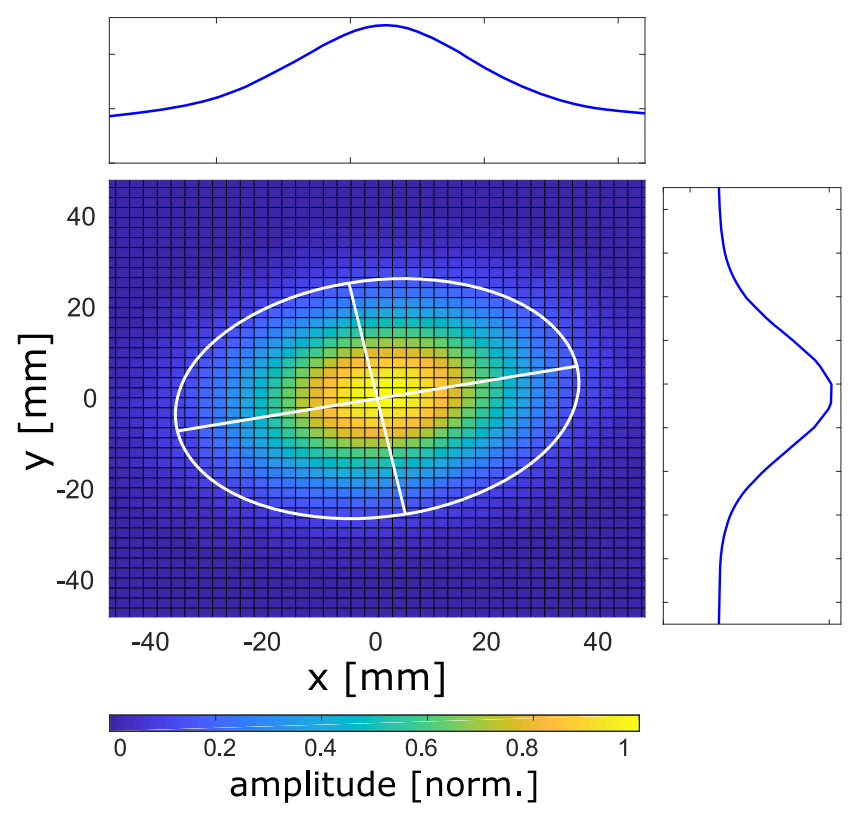

FIG. 6. Measured 2D beam profile at $f_{0}=103.14 \mathrm{GHz}$ (distance to antenna aperture $d z=410 \mathrm{~mm}$ ).

The inset figure shows Doppler spectrum for very low scattered power at $f_{0}=86.25 \mathrm{GHz}(t=1750 \mathrm{~ms})$ to demonstrate the sufficient resolution of the Doppler peak and the narrow contribution of the specular reflection around zero frequency. The solid black indicates the estimated Doppler frequency, which has been determined by fitting a single Gaussian to the Doppler spectrum. The backscattered power $P \sim S\left(k_{\perp}\right) \sim|\delta n|^{2}$ is given by the area beneath the fitted Doppler peak. ${ }^{9}$ The measured scattered power is shown in Fig. 4(e) as a blue line. As expected, the scattered power increases with $1 / \mid k_{\perp} l$, but for $\left|k_{\perp}\right| \lesssim 5 \mathrm{~cm}^{-1}$, the power decreases. This is caused by two effects: First, the PAA characteristic causes an attenuation at small $\theta$ [cf. Fig. 2(b)]. If this calibration factor is taken into account the power decrease for $\left|k_{\perp}\right| \lesssim 5 \mathrm{~cm}^{-1}$ is partly compensated as indicated in Fig. 5(e) (red line). Second, the scattered power decreases significantly if $R=\left|k_{\|} / k_{\perp}\right|>0.1 .^{5}$ Since the measurement plane of the PAA is fixed, $R$ can vary significantly during the steering sweep because the geometrical contribution of the parallel refractive index increases with $|\theta|$, as indicated in Fig. 7(a). The ratio $R$ is depicted in Fig. 7(b). Except for the singularity at normal incidence $\left(\theta=-5.5^{\circ}\right)$, $R \approx 0.1$. The wavenumber spectrum $S\left(k_{\perp}\right)$ is shown separate for positive and negative $\theta$ as red and blue dots in Fig. 7(c). For large wavenumbers, both spectra exhibit a power law decay with $k^{-9 / 2}$. Similar decay rates are also reported from other steered $X$-mode Doppler systems. ${ }^{9}$ At intermediate wavenumbers $k_{\perp}<5-11 \mathrm{~cm}^{-1}$, differences in the spectral shape are observed, which could be attributed to the different runs of $R$ for $|\theta|<10^{\circ}$. For $k_{\perp}=6-14 \mathrm{~cm}^{-1}$, the spectrum scales approximately as $k^{-3 / 2}$. The observed scaling regions agree in principle with predictions for two-dimensional uid turbulence (dual cascade). ${ }^{8}$ The transition between the scaling regions is determined by the energy input from driving instabilities. For drift-type instabilities with maximum linear growth rates $k_{\perp} \rho_{s} \approx 1$ ( $\rho_{s}$ being the ion gyroradius at electron temperature $T_{e}$ ), this wavenumber range is indicated in Fig. 7(c) for
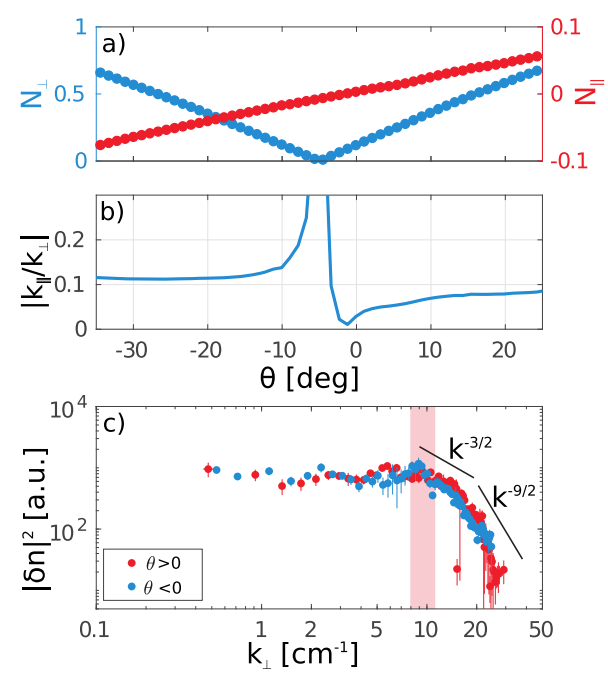

FIG. 7. Evolution of $N_{\perp}$ and $N_{\|}$(a) and the ratio $R$ (b) with steering angle $\theta$. (c) Fluctuation wavenumber spectra derived from PAA measurement (see text for details).

typical near-SOL electron temperatures $T_{e}=50-100 \mathrm{eV}$ as a shaded box.

\section{SUMMARY AND CONCLUSION}

A bistatic phased array Doppler reflectometer system operating in W-band with $X$-mode polarization has recently been installed at the stellarator W7-X. The microwave beam can be steered reliable in the measurement plane perpendicular to the local magnetic field in the range $-25^{\circ} \leq \theta \leq 25^{\circ}$ with good beam quality but low power (total coupling efficiency $\sim-15 \mathrm{~dB}$ ). The measured $I Q$-signal has a low signal-to-noise ratio $\left(P_{S / N} \approx 12 \mathrm{~dB}\right)$, but the Doppler peak is sufficiently resolved. A first attempt has been made to derive a fluctuation wavenumber spectrum $S\left(k_{\perp}\right)$ from the measured backscattered power. The spectrum exhibits two distinct scaling regions $\left(k_{\perp}^{3 / 2}\right.$ and $k_{\perp}^{9 / 2}$ ), which are reasonable. Similar high- $k_{\perp}$ power law decay rates have been reported from other steerable Doppler systems. ${ }^{9}$ In the derivation of $S\left(k_{\perp}\right)$, many assumptions must be made which need a more detailed analysis. For example, it has been assumed that the diagnostic response for all steering angles scales linear with the fluctuation amplitude. Especially for $X$-mode polarization, a saturation of the measured scattered power for larger fluctuation amplitudes appears. ${ }^{9,13}$ Since the threshold depends on $k_{\perp}$ or equivalently on the steering angle $\theta$, it can significantly flatten the spectrum at small scales.

\section{ACKNOWLEDGMENTS}

This work was also performed within the framework of the Helmholtz Virtual Institute on Plasma Dynamical Processes and Turbulence Studies using Advanced Microwave Diagnostics.

This work has been carried out within the framework of the EUROfusion Consortium and has received funding from the Euratom research and training programme 2014-2018 under Grant Agreement No. 633053. The views and opinions expressed herein do not necessarily reflect those of the European Commission. 
${ }^{1}$ G. D. Conway, J. Schirmer, S. Klenge, and E. W. Suttrop, "Holzhauer and the ASDEX upgrade team," Plasma Phys. Controlled Fusion 46, 951 (2004).

${ }^{2}$ A. J. Fenn, D. H. Temme, W. P. Delaney, and W. E. Courtney, Lincoln Lab. J. 12, 321 (2000).

${ }^{3}$ T. Happel, T. Estrada, E. Blanco, V. Tribaldos, A. Cappa, and A. Bustos, Rev. Sci. Instrum. 80, 073502 (2009).

${ }^{4}$ P. Hennequin, C. Honoré, A. Truc, A. Quéméneur, C. Fenzi-Bonizec, C. Bourdelle et al., Nucl. Fusion 46, S771 (2006).

${ }^{5}$ J. C. Hillesheim, N. A. Crocker, W. A. Peebles, H. Meyer, A. Meakins, A. R. Field et al., Nucl. Fusion 55, 073024 (2015).

${ }^{6}$ M. Hirsch, E. Holzhauer, J. Baldzuhn, B. Kurzan, and B. Scott, Plasma Phys. Controlled Fusion 43, 1641 (2001).

${ }^{7}$ T. Klinger, A. Alonso, S. A. Bozhenkov, R. Burhenn, A. Dinklage, G. Fuchert et al., Plasma Phys. Controlled Fusion 59, 014018 (2017).

${ }^{8}$ R. H. Kraichnan, Phys. Fluids 10, 1417 (1967).

${ }^{9}$ C. Lechte, G. D. Conway, T. Görler, C. Tröster-Schmid, and ASDEX Upgrade Team, Plasma Phys. Controlled Fusion 59, 075006 (2017).

${ }^{10}$ Y. Lin, R. Nazikian, J. H. Irby, and E. S. Marmar, Plasma Phys. Controlled Fusion 43, L1-L8 (2001).
${ }^{11}$ N. B. Marushchenko, Y. Turkin, and H. Maassberg, Comput. Phys. Commun. 185, 165 (2014).

${ }^{12}$ E. Pasch, M. N. A. Beurskens, S. A. Bozhenkov, G. Fuchert, J. Knauer, R. C. Wolf et al., Rev. Sci. Instrum. 87, 11E729 (2016).

${ }^{13}$ J. R. Pinzón, T. Happel, E. Blanco, G. D. Conway, T. Estrada, and U. Stroth, Plasma Phys. Controlled Fusion 59, 035005 (2017).

${ }^{14}$ P. Rohmann, S. Wolf, W. Kasparek, B. Plaum, and J. Hesselbarth, in IEEE International Symposium on Phased Array Systems and Technology (IEEE, 2013), Vol. 559.

${ }^{15}$ J. T. Williams, P. Baccarelli, S. Paulotto, and D. R. Jackson, IEEE Trans. Antennas Propag. 614484 (2013).

${ }^{16}$ T. Windisch, T. Estrada, M. Hirsch, W. Kasparek, A. Krämer-Flecken, C. Lechte et al., 42nd EPS Conference on Plasma Physics (European Physical Society, 2016), P1.163.

${ }^{17}$ T. Windisch, A. Krämer-Flecken, J. L. Velasco, A. Könies, C. Nührenberg, O. Grulke et al, Plasma Phys. Controlled Fusion 59, 105002 (2017).

${ }^{18} \mathrm{~S}$. Wolf, P. Rohmann, W. Kasparek, B. Plaum, and C. Lechte, in Proceedings of the 11th International Reflectometry Workshop, Palaiseau, 2013, http://www.aug.ipp.mpg.de/IRW/.

${ }^{19} \mathrm{~S}$. Wolf, "Development of a phased array antenna for Doppler reflectometry in fusion devices," Ph.D. thesis, University Stuittgart. 